Implementation of smoke-free policy in university hospital decreases carboxyhemoglobin level in inpatients undergoing surgery. Anesthesiology 2007; 106: 406-7.

4 Warner DO, Sarr MG, Offord KP, Dale LC.

Anesthesiologists, general surgeons, and tobacco interventions in the perioperative period. Anesth Analg 2004; 99: 1766-73.

\section{Survival with extreme lactic acidosis following ethylene glycol poisoning?}

To the Editor:

A 47-yr-old woman, with a past history of self-poisonings associated with a bipolar disorder, was found comatose by the relatives who suspected a drug overdose. The time delay from ingestion to presentation was approximately $12 \mathrm{hr}$. The initial Glasgow coma scale was 3 and the patient's trachea was intubated on the scene. On arrival in the emergency department $(1300 \mathrm{hr})$, vital signs were as follows: heart rate, 85 beats. $\mathrm{min}^{-1}$, arterial blood pressure, $124 / 61 \mathrm{mmHg}$, and rectal temperature, $30.5^{\circ} \mathrm{C}$. The neurological examination revealed fixed and dilated pupils, and the deep tendon reflexes were either depressed or absent. Laboratory data revealed a profound metabolic acidosis with an arterial blood $\mathrm{pH}$ of 6.56 and a lactate level of $60 \mathrm{mmol} \cdot \mathrm{L}^{-1}(\mathrm{~N}<1.3)$, while bicarbonate was $4.5 \mathrm{mmol} \cdot \mathrm{L}^{-1}$ and $\mathrm{pCO}_{2} 35 \mathrm{mmHg}$ (under mechanical ventilation, with no correction for temperature). This determination had been performed on a Radiometer $\mathrm{ABL} 725^{\mathrm{TM}}$ blood gas analyzer (Radiometer A/S, Bronshoj, Denmark). Despite this remarkable hyperlactatemia, the calculated anion gap was $35.7 \mathrm{mmol} \cdot \mathrm{L}^{-1}$. Blood urea was $13.5 \mathrm{mmol} \cdot \mathrm{L}^{-1}$ and the glucose concentration was $8.1 \mathrm{mmol} \cdot \mathrm{L}^{-1}$. The estimated osmol gap was $90 \mathrm{mOsm} \cdot \mathrm{kg}^{-1}$. The serum creatinine concentration was within normal range (132 $\mu \mathrm{mol} \cdot \mathrm{L}^{-1}$ ), but the patient was oliguric from the time of admission. Central venous blood oxygen saturation remained above $65 \%$.

A toxic alcohol ingestion was suspected, and oxalate crystals were detected in the urine sample (oxaluria $184 \mathrm{mg} \cdot \mathrm{g}^{-1}$ creatinine; $\mathrm{N}<32$ ). The diagnosis of ethylene glycol (EG) poisoning was confirmed in the serum $\left(2.78 \mathrm{~g} \cdot \mathrm{L}^{-1}\right)$. No other drug was detected by the toxicological screen. Due to the severity of metabolic acidosis with oliguria despite fluid replacement, hemodialysis was started in the intensive care unit (ICU) for a total duration of eight hours, and ethanol was preferred over fomepizole as antidotal treatment. We observed some discrepancy between the rapid correction of acidemia and the persistence of high
TABLE I Biological data from the time of admission. Uncorrected lactate measurements by Radiometer ABL $725^{\mathrm{TM}}$ (corrected for $37^{\circ} \mathrm{C}$ body temperature).

\begin{tabular}{llllll}
\hline Time & $1300 \mathrm{hr}$ & $1800 \mathrm{hr}$ & $1830 \mathrm{hr}$ & $2330 \mathrm{hr}$ & $0200 \mathrm{hr}$ \\
\hline Body temperature $\left({ }^{\circ} \mathrm{C}\right)$ & 30.5 & 36.9 & 37.1 & 36.9 & 37.2 \\
$\mathrm{pH}$ & 6.56 & 7.37 & 7.39 & 7.35 & 7.38 \\
Lactate $\left(\mathrm{mmol} \cdot \mathrm{L}^{-1}\right)$ & 60 & 25 & 15 & 7.8 & 5.2 \\
$\begin{array}{l}\text { Base excess }\left(\mathrm{mmol} \cdot \mathrm{L}^{-1}\right) \\
\text { Bicarbonate }\left(\mathrm{mmol} \cdot \mathrm{L}^{-1}\right)\end{array}$ & 3 & 11 & 14 & 18 & 20 \\
$\begin{array}{l}\text { Ethylene glycol }\left(\mathrm{g} \cdot \mathrm{L}^{-1}\right) \\
\text { Ethanol }\left(\mathrm{g} \cdot \mathrm{L}^{-1}\right)\end{array}$ & 2.78 & 1.0 & 0.57 & - & - \\
$\begin{array}{l}\text { Anion gap }(16 \pm 4) \\
\left(\text { mmol } \cdot \mathrm{L}^{-1}\right)\end{array}$ & 36.2 & 32.5 & 19.9 & - & 13.2 \\
$\begin{array}{l}\text { Osmolar gap } \\
\left(\mathrm{mOsm}_{\mathrm{kgg}} \mathrm{kg}^{-1}\right)\end{array}$ & 90.0 & 37.5 & 25.5 & 19.7 & 13.9 \\
\hline
\end{tabular}

TABLE II Analyzers for which interference on lactate results have been described for both glycolic acid and glyoxylic acid (italics) or glyoxylic acid alone. ${ }^{6}$

Synchron LX 20 (Beckman Coulter)

Bayer 860 (Bayer Diagnostics)

ABL 625, 725, 825 (Radiometer)

OMNI S (Roche Diagnostics)

CCX 4 (Nova)

Chiron 865 (Bayer Diagnostics)

Rapid Lab 1265 (Siemens Diagnostics)

Architect ci 8200 (Abbott)

serum lactate values (Table I). Ethylene glycol was no longer detectable in the serum at the end of hemodialysis. The patient became fully awake $12 \mathrm{hr}$ following admission, and her trachea was extubated shortly thereafter. She did not present immediate or delayed neurological sequelae. Intermittent hemodialysis was still required after ICU discharge for a period of five weeks, but the patient ultimately recovered normal renal function.

Severe metabolic acidosis is a common feature of toxic alcohol poisoning when the patient presents with a delay from the time of ingestion. For both methanol and EG poisoning, the final prognosis is clearly related to the severity of the initial acidosis. ${ }^{1}$ However, with aggressive supportive care, adequate antidotal therapy, and extrarenal elimination techniques when indicated, complete recovery is still possible, despite extremely low $\mathrm{pH}$ values. Fomepizole is an extremely potent inhibitor of alcohol dehydrogenase, and is effective for treatment of EG poisoning, with minimal adverse effects. The main reason fomepizole is not used in many hospitals pertains to the high acquisition cost and limited availability of this drug. Maximum therapeutic benefit can be achieved by administering 


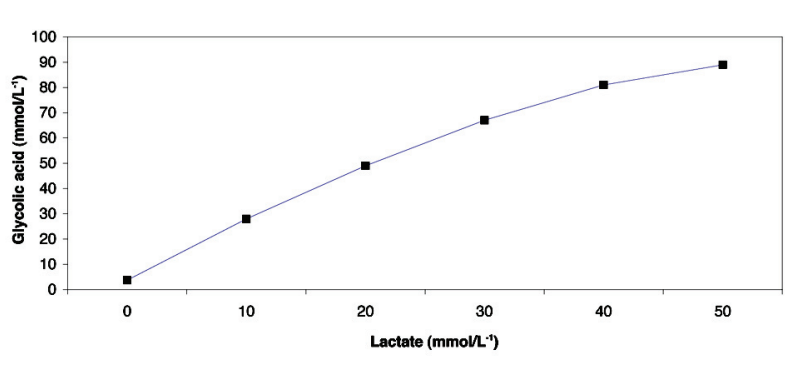

FIGURE Whole blood spiked with increasing concentrations of glycolic acid and analyzed for lactate concentration on the Radiometer ABL $725^{\mathrm{TM}}$.

fomepizole before the onset of renal failure and severe metabolic acidosis, as fomepizole can be used without hemodialysis as blocking therapy. ${ }^{2}$

Blakeley et $a l^{3}$ described a case of EG poisoning where the patient survived with a blood $\mathrm{pH}$ measured at 6.46. It is generally accepted that the metabolic acidosis is mainly due to the accumulation of toxic metabolites. For EG poisoning, there is an inverse relationship between the serum bicarbonate level and the concentration of glycolic acid, the major metabolite of EG. ${ }^{1}$ Lactic acidosis following toxic alcohol poisoning is usually mild and often associated with hemodynamic instability. It is usually seen as a terminal event. The initial lactate level in our patient was surprisingly high and did not fit with the anion gap calculation. Recent reports describe false lactate elevation with several arterial blood gas analyzers ${ }^{4-6}$ (Table II). We confirm that an interference between glycolic acid and lactate reading exists, at least for the Radiometer ABL $725^{\mathrm{TM}}$ (amperometric electrodes with enzymatic membranes). Indeed, by spiking whole blood samples with increasing glycolic acid concentrations from 0 to $50 \mathrm{mmol} \cdot \mathrm{L}^{-1}$, we observed a parallel, rather than a strictly linear, increase of lactate concentrations (Figure). When the patient's initial blood sample was re-analyzed on a Unicell DxI 800 (Beckman-Coulter, Fullerton, CA, USA) using a method based on a lactate oxidase/peroxidase-coupled reaction with endpoint determination, a valid result could not be obtained for the lactate concentration. By spiking aqueous solutions with glycolic acid concentrations from 0 to $10 \mathrm{mmol} \cdot \mathrm{L}^{-1}$, we obtained apparent lactate values up to $8 \mathrm{mmol} \cdot \mathrm{L}^{-1}$ of glycolic acid, whereas higher concentrations produced a "rate high" error flag. At a concentration of $8 \mathrm{mmol} \cdot \mathrm{L}^{-1}$, glycolic acid induced an increase of $0.4 \mathrm{mmol} \cdot \mathrm{L}^{-1}$ for the lactate results. The cause of interference is likely an incomplete specificity of the enzyme L-lactate oxidase used in some analyzers. The L-lactate concentration is computed from the quantification (either by amperometric or colorimetric methods) of the hydrogen peroxide concentration resulting from the enzymatic reaction between L-lactate and oxygen.

In conclusion, this case illustrates that survival is possible despite extreme metabolic acidosis following EG poisoning, but that the interpretation of high lactate concentrations should be made cautiously with a majority of arterial blood gas analyzers commonly used in emergency departments. A false hyperlactatemia could lead to an erroneous prognosis. The absence of cardiocirculatory failure, together with a high central venous oxygen saturation, should alert clinicians of this possible interference.

Diego Castanares-Zapatero MD

Catherine Fillée MD

Marianne Philippe MD PhD

Philippe Hantson MD PhD

Cliniques St-Luc, Université catholique de Louvain, Brussels, Belgium

E-mail: philippe.hantson@uclouvain.be

Accepted for publication February 20, 2008.

\section{References}

1 Hylander B, Kjellstrand CM. Prognostic factors and treatment of severe ethylene glycol intoxication. Intensive Care Med 1996; 22: 546-52.

2 Velez LI, Shepherd G, Lee YC, Keyes DC. Ethylene glycol ingestion treated only with fomepizole. J Med Toxicol 2007; 3: 125-8.

3 Blakeley KR, Rinner SE, Knochel JP. Survival of ethylene glycol poisoning with profound acidemia, New Engl J Med 1993; 328: 515-6.

4 Fijen JW, Kemperman H, Ververs FF, Meulenbelt J. False hyperlactatemia in ethylene glycol poisoning. Intensive Care Med 2006; 32: 626-7.

5 Brindley PG, Butler MS, Cembrowski G, Brindley DN. Falsely elevated point-of-care lactate measurement after ingestion of ethylene glycol. CMAJ 2007; 176: 10979.

6 Graine H, Toumi K, Roullier V, Capean J, Lefevre $G$. Interference of ethylene glycol on lactate assays (French). Ann Biol Clin (Paris) 2007; 65: 421-4.

\section{Another failure mechanism leading to patient-controlled analgesia overdoses}

To the Editor:

Patient-controlled analgesia (PCA) is used extensively to treat postoperative pain. Potential benefits include 